\title{
Dynamical and Microrheological Analysis of Amyloplasts in the Plant Root Gravity-Sensing Cells
}

\author{
Zhongyu Zheng $^{2} \cdot$ Junjie Zou $^{1} \cdot$ Hanhai $\mathrm{Li}^{2} \cdot$ Shan Xue $^{1} \cdot$ Jie Le $^{1} \cdot$ Yuren Wang $^{2}$
}

Received: 7 February 2015 / Accepted: 3 July 2015 / Published online: 4 August 2015

(C) Springer Science+Business Media Dordrecht 2015

\begin{abstract}
Gravitropism in plants is one of the most controversial issues. In the most wildly accepted starch-statolith hypothesis the sedimentation movement of amyloplasts in the gravisensing columella cells primarily triggers the asymmetric distribution of auxin which leads to the differential growth of the plant root. It has been gradually recognized that the inhomogeneous structures in statocytes arising from intracellular components such as cytoskeletons significantly affect the complex movements of amyloplasts and the final gravimorphogenesis. In this letter, we implement a diffusive dynamics measurement and inplanta microrheological analysis of amyloplasts in the wild-type plants and actin cytoskeleton mutants for the first time. We found that the intracellular environment of columella cells exhibits the
\end{abstract}

\author{
Zhongyu Zheng \\ zzy@imech.ac.cn \\ Junjie Zou \\ zoujunjie@ibcas.ac.cn \\ Hanhai Li \\ lihanhai@imech.ac.cn \\ Shan Xue \\ xueshan1984@yeah.net \\ Jie Le \\ lejie@ibcas.ac.cn \\ Yuren Wang \\ yurenwang@imech.ac.cn
}

1 Key Laboratory of Plant Molecular Physiology, Institute of Botany, Chinese Academy of Sciences, Beijing 100093, China

2 Key Laboratory of Microgravity, Institute of Mechanics, Chinese Academy of Sciences, Beijing 100190, China spatial heterogeneity and the cage-confinement on amyloplasts which is the typically characteristics in colloidal suspensions. By comparing the distinct diffusive dynamics of amyloplasts in different types of plants with the behaviors of colloidal systems in different states, we quantitatively characterized the influence of the actin organization dominated intracellular envoronments on the amyloplast movements. Furthermore, the cage-confinement strength was measured by calculating the spatial fluctuation of local apparent viscosity within the columella cells. Finally, a linear association between the initial mechanical stimulation in the columella cells the subsequent intercellular signal transduction and the final gravity response was observed and a possible gravity sensing mechanism was suggested. It suggests the existence of a potential gravity-sensing mechanism that dictates a linear frustration effect of the actin cytoskeleton on the conversion of the mechanical stimulation of amyloplasts into gravitropic signals.

Keywords Plant gravitropism - Gravity sensing · Amyloplasts · Columella cells · Microrheology · Diffusion $\cdot$ Cage-confinement

\section{Introduction}

Over a century ago, Darwin described the opposite growth directions of roots and shoots in his book 'The power of movement in plants'. Gravitropism is vital for plant growth and morphogenesis. It enables the downward growth of roots to efficiently acquire water and nutrients while optimize the upright growth of the shoot systems to absorb sufficient light and $\mathrm{CO}_{2}$ for enhanced photosynthesis (Blancaflor 2013). Gravitropism contains three major phases: gravity sensing, signal transduction and gravity 
response (Fig. 1). As the primary phase of gravitropism, plant gravity sensing has been intensively investigated and the starch-statolith hypothesis is most widely accepted. The statoliths, generally existing in animals, are vital for the sense of balance and the response to gravity, e.g. statoliths in statocytes (sensing cells) for invertebrates and in inner ear for vertebrates. In plants, starchy-amyloplasts in columella cells play the role as statoliths in root gravisensing (Morita 2010). The sedimentation movement of the amyloplasts is essential for gravity sensing by converting the gravitational potential energy into a biochemical signal (Leitz et al. 2009) primarily triggering auxin asymmetric distribution, and leading to differential growth across the organ during the gravitropic response (Vanneste and Auxin 2009) (Fig. 1).

Actually, the amyloplast movements are the superposition of the directional sedimentation imposed by external gravity force and the random Brownian motion influenced by inherent environment of columella cells. The Latrunclin B (Lat B) treatment, which induces the depolymerization of F-actin networks, has been reported to provide an enhanced effect on the sedimentation of amyloplasts in the living columella cells of Arabidopsis (Hou et al. 2004). It indicates a probably significant influence of actin organization in determining the intracellular microenvironment, the amyloplast sedimentation process and the final gravity response. The conversion process from gravity force stimulation to biochemical signal requires the interaction between amyloplasts and the surrounding components within the columella cells. Two hypothetical models for the gravity sensing mechanisms have been proposed. In one model, the amyloplasts are supposed to open the stretchactivated $\mathrm{Ca}^{2+}$ channels (SACs) by exerting tension on the actin filament or on bridging filaments that link SACs (Perbal and Driss-Ecole 2003). In the other one, it has been found recently that the impact of amyloplasts on the deformation of the endoplasmic reticulum (ER) membranes might potentially activate mechanosensitive ion channels (Leitz et al. 2009) It has been gradually recognized that the interaction with surrounding intracellular components, such as the cytoskeleton and vacuole, greatly affects the
Brownian diffusion of amyloplasts and induces the complexity of amyloplast movement (Hou et al. 2004; Perbal and Driss-Ecole 2003). The sedimentation rate, velocity and number of amyloplasts were frequently studied (Leitz et al. 2009; Perbal and Driss-Ecole 2003; Toyota et al. 2013; Palmieri and Kiss 2005; Swarup et al. 2005). However, due to such complex intracellular environment, these average measurements can hardly detect the essential interactions between the amyloplasts and intracellular components (e.g. F-actin networks or ER networks) and identify the exact loading sites and loading modes for the gravity force sensing. The diffusive dynamics of a single particle is a reflection of the interaction with the surrounding local environments which largely influences the collective sedimentation movements (Squires and Mason 2009). Hence, a quantified measurement of the diffusive dynamics and collective movements of amyloplasts is required to clarify the interaction with actin cytoskeleton and other unknown components. It is very important for understanding how the kinetic mechanical energy transforms to biochemical signals, which determines the final gravity response. However, the diffusive dynamics and complex movements of amyloplasts are still poorly understood. In this work, we quantitatively analyzed the diffusive dynamics of amyloplasts during sedimentation from the single-particle tracking, movement trajectories and mean square displacement (MSD) measurement. We focused on the influence of the actin organization, hence the wild type plants (Arabidopsis ecotype Columbia, Col), dis1 plants with the mutation of the DISTORTEDI (DIS1) gene and the Lat B treated Col. and dis 1 plants were studied, respectively. The intracellular environments of the gravisensing collumelar cells in col., dis1 and Lat B treated plants behave as highly dense, jammed and dilute colloidal suspensions.

The intracellular structural heterogeneity and cageconfinement effect can be quantitatively characterized by measuring the local viscosity through the microrheological methodology. In the past decade, microrheology (Crocker et al. 2000; Levine and Lubensky 2000) has been rapidly developed as a successful method to explore the relation between local mechanical properties and local structure in
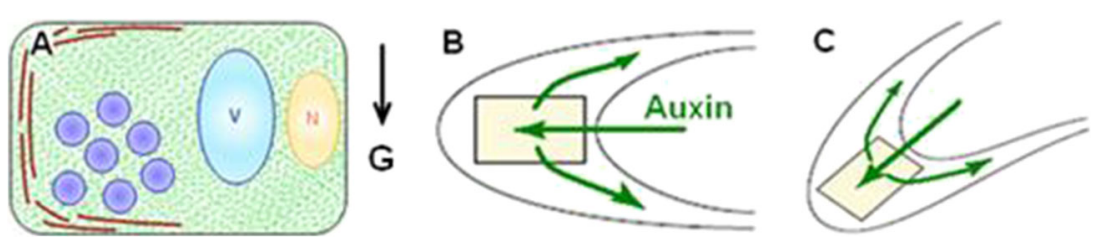

Fig. 1 Cartoons describing the three phases for plant root gravitropism a Sedimentation of amyloplasts in the columella cell. ' $N$ ' and ' $V$ ' denote the nucleus and the vacuole. The purple spheres denote the amyloplasts. The green dots and brown curves denote the F-actin networks and the endoplasmic reticulum. b The redistribution of auxin across the root cap. The green arrows denote the auxin flow which is larger in the lower side of root cap than that in the upper side. $\mathbf{c}$ The downward curvature of the plant root 
complex fluids (Squires and Mason 2009; Crocker et al. 2000; Levine and Lubensky 2000; Waigh 2005; Hunter and Weeks 2012; Wirtz 2009; Mizuno et al. 2007; Chaudhuri et al. 2007). Microrheology analyses have been applied in vitro to quantitatively evaluate the mechanical functions of the cytoskeletal network during cell locomotion and cell division (Wirtz 2009; Mizuno et al. 2007; Chaudhuri et al. 2007). Usually, microprobes such as colloidal spheres are needed to inject into the modeling biosystems (Mizuno et al. 2007; Chaudhuri et al. 2007).

Here, we developed an intravital microrheology measurement in the columella cells by using the amyloplasts as native micro-probes. The spatial distribution of apparent viscosity was obtained from measuring the short-time diffusion coefficients of amyloplasts. We found that the spatial fluctuation of the local apparent viscosity in the columella cells in the wild type plants is only about $20 \%$ of that in the dis1 plants, but two times larger than that in the Lat B treated plants. These results are consistent with the observation of diffusive dynamics of amyloplasts. Some new insight into gravity sensing was proposed by comparing mechanical stimulation with the resulting biochemical signal and gravitational response.

\section{Materials and Methods}

\section{Plant Materials and Growth}

Plant seeds of wild-type Arabidopsis thaliana ecotype Col and dis 1 were surface sterilized and placed in the Petri dishes containing $1 / 2$ Mannitol salt (MS) agar $(0.8 \%$, w/v) and incubated in the $4{ }^{\circ} \mathrm{C}$ for $2 \mathrm{~d}$. Then the plates were put in the growth chamber under a $16 \mathrm{~h}$-light/8h-dark photoperiod at $22{ }^{\circ} \mathrm{C}$ for 4 days

\section{Lat B Treatments}

Lat B treatment was conducted as the method described before Hou et al. (2004). Arabidopsis seeds were surface sterilized and placed in the Petri dishes containing $1 / 2$ Murashige and Skoog agar $(0.8 \%$, w/v) medium and incubated for 2 days at $4{ }^{\circ} \mathrm{C}$. Then the Petri dishes were put vertically and the seedling were grown at $22{ }^{\circ} \mathrm{C}$ and under 16-hour daily light per day. After 4-day growth, $200 \mathrm{nM}$ Lat B solution was added into Petri dishes which containing seedlings. After $1 \mathrm{~h}$ treatment, the Lat B solution was removed and the Petri dishes were kept vertically for additional $30 \mathrm{~min}$ before the dishes were reoriented at 90 degrees. The photographs were taken at indicated time and the curvature of roots was measured using imageJ software (image J 1.49n).

\section{Amyloplast Observation}

Sample preparation was conducted as described before Leitz et al. (2009). The plant roots were imaged by DIC by using a light microscope Olympus BX51 (DP25, DP70). The microscope was lied down and a rotatable disc was used to mount the slides. The images were captured before and after 90 degree reorientation.

\section{Rotary Vertical Stage Microscopy}

To image the amyloplast sedimentation in the columella cells, we developed a vertical confocal microscopy. An inverted phase microscope was fixed horizontally on a custom-made steel support. A rotary sample stage was mounted on the optical table with the optical path passing through its center of rotation. We initially mounted the plant roots vertically and made the columella cells right fall inside the field of view. When the plant roots were reoriented horizontally by rotation the sample stage, the amyloplast sedimentation was imaged by applying differential interference contrast (DIC) mode and recorded by a charge coupled device (CCD).

\section{Mean-Square-Displacement (MSD) of Amyloplasts}

The amyloplast sedimentation trajectories were obtained at 1 frame per second by microscopy recording and image processing in IDL. The diffusive motion ofamyloplasts during gravity sedimentation was analyzed by measuring the mean-square-displacements: $M S D_{x, y}(t)=\left\langle\Delta r_{x, y}(t)^{2}\right\rangle-$ $\left\langle\Delta r_{x, y}(t)\right\rangle^{2}=2 D_{\mathrm{x}, \mathrm{y}} t$, where $\Delta r_{x, y}(t)$ is the displacements of an amyloplast in the horizontal (x) and gravitational (y) directions during time interval $t, \mathrm{D}$ is the diffusion coefficient and \langle\rangle is the ensemble average (Crocker et al. 2000; Marcus et al. 1999). The sedimentation movement has been subtracted in the second term. The MSD(t) calculated by averaging for all amyloplasts is the same as that calculated by averaging over all the subregions within an error of $10 \%$.

\section{Apparent Local Viscosity}

According to the fluctuation-dissipation theory, the Brownian motion of a micro-particle is driven by the random fluctuation of surrounding fluid with thermal energy $k_{B} T$, where $k_{B}$ is the Boltzmann's constant and $T$ is the temperature and is also depressed by the friction of surrounding fluid with viscosity $\eta$ (Squires and Mason 2009). By using the Stokes-Einstein relation, the apparent local viscosity $\eta$ in each subcellular region was measured from the short-time diffusion coefficient $D=k_{B} T /(6 \pi R \eta)$ where $k_{B}$ is the Boltzmann constant, $T$ is the temperature 
and $R$ is the amyloplast radius (here $R=2.0 \mu \mathrm{m}$ ) (Blancaflor 2013; Morita 2010; Leitz et al. 2009; Vanneste and Auxin 2009; Hou et al. 2004; Perbal and Driss-Ecole 2003; Toyota et al. 2013; Palmieri and Kiss 2005; Swarup et al. 2005; Squires and Mason 2009; Crocker et al. 2000; Levine and Lubensky 2000). We analyzed the Brownian motion and collective motion by measuring the short-time Brownian diffusion and intermediate-time caged diffusion of each amyloplasts during their sedimentation process.

\section{Root Curvature Angle}

To measure the curvature angle of the plant roots, we first put the agar substrate vertically and made the plants horizontally. Then the growing process of the plants was recorded by a digital camera at 1 frame every 10 minutes for more than 10 hours. The curvature angle of the plant root was measured as the angle between the plant root and the horizontal direction by using imageJ software (imageJ $1.49 n)$

\section{DII-VENUS Florescence Intensity Measurement}

Four-day-old DII-VENUS or dis $1 x$ DII-VENUS seedlings grown on each Petri plate containing 1/2 MS medium were transplanted onto a new plate. After 2 hours, the plates were reoriented 90 degrees from the vertical direction to the horizontal direction. Then the the seedlings were mounted, and the fluorescence intensity of DII-VENUS were observed using a confocal laser scanning microscope (Olympus, Tokyo, Japan, FV1000-MPE) and recorded at the indicated time by a CCD. For visualization of the root cell organization, the roots were stained with propidium iodide (Sigma). We measured the DII-VENUS fluorescence intensity in the root tips as the method described before Brunoud et al. (2012). To quantified the fluorescence intensity of nuclei in the cells, 3 to 4 optical sections were obtained and projected. The fluorescence intensity of nuclei (about 8 Later root cells from LRC2 to LRC4) (Band et al. 2014) was extracted at each time point using imageJ software (imageJ $1.49 n$ ) and the values were analyzed using SigmaPlot 12.0.

\section{Results and Discussion}

The Brownian dynamics of amyloplasts should be significantly influenced by the interaction with the highly inhomogeneous intracellular environment induced by the various cytoplasmic organoids, which leads to a complex movement of amyloplasts during sedimentation in the gravisensing cells (Blancaflor 2013; Leitz et al. 2009; Hou et al. 2004) To identify the dominant influence of actin cytoskeleton on the structural heterogeneity and amyloplast movements within the columella cells, we studied the dynamics and micorheology of amyloplasts during sedimentation in wild type, the actin related protein $A R P 3$ mutant dis 1 and the Lat $\mathrm{B}$ treated plants. We analyzed the distinctly dynamical and microrheological behaviors of amyloplasts in these four types of plants by comparison with that in the colloidal suspensions from the respects of sedimentation time, movement trajectories, MSDs, diffusivities and apparent viscosities.

\section{Movement Trajectories and Cage-Effect During Amyloplast Sedimentation}

First we directly visualize the amyloplast sedimentation in central columella cells.After $90^{\circ}$ degree reorientation of the plant, most of the amyloplasts fell onto the new bottom cell wall usually within $400 \mathrm{~s}, 600 \mathrm{~s}$ and $300 \mathrm{~s}$ in the wild type, dis1 mutant and Lat B treated plants, respectively Mutation of the DISTORTEDI (DIS1) gene, which encodes the Actin Related Protein 3 (ARP3) subunit of ARP2/3 complex, induces the formation of mis-organized thick actin bundles in the trichome compared to wildtype plants (Le et al. 2003; Brunoud et al. 2012; Band et al. 2014). ARP2/3 complex is an evolutionarily conserved seven-subunit multiprotein complex, and is an actin filament-nucleating machine that binds to the sides of existing actin filaments and nucleates new filaments at a distinctive 70 degree angle (Basu et al. 2005). Lat B treatment is well known to be able to disrupt the F-actin networks in the columella cells (Hou et al. 2004). The enhanced and reduced sedimentation rates of amyloplasts in Lat B treated plants and untreated dis1 mutants are consistent with the disrupted F-actin networks and F-actin bundles in the columella cells. We next analyzed the diffusion and collective motion of amyloplasts from their typical trajectories obtained from single-particle tracking (Fig. 2a-d). Here, the mean sedimentation of all amyplasts in one columella cell was subtracted to highlight the diffusive motion. The amyloplasts in the wild type plants show a chainlike pattern with frequent small-step rattling motion within small regions and occasional large-step motion between these regions (Fig. 2a). This is the typical characteristics of a cage-effect generally existing in complex fluids such as dense colloidal suspensions (Fig. 3a, c) (Hunter and Weeks 2012; Marcus et al. 1999; Zheng et al. 2011; Zheng et al. 2014; Zheng and Han 2010). The localized small-step rattling motion corresponds to the confined random Brownian motion within a cage while the chain-like large-step motion corresponds to the cooperative escape from the cages. These results suggest the colloidal-like spatial heterogeneities within Col columella cells and the facilitation of amyloplast sedimentation through a series of intermittent collective cage-escape processes. The typical trajectories of amyloplasts in disl and Lat B treated 
Fig. 2 Movement trajectories of amyloplasts in the central columella cells after 90 degree reorientation. The solid and open circles denote the start and the end point of each amyloplast. A single columella cell at the second layer was chosen to show exactly the movement trajectories of amyloplasts for each type of plant

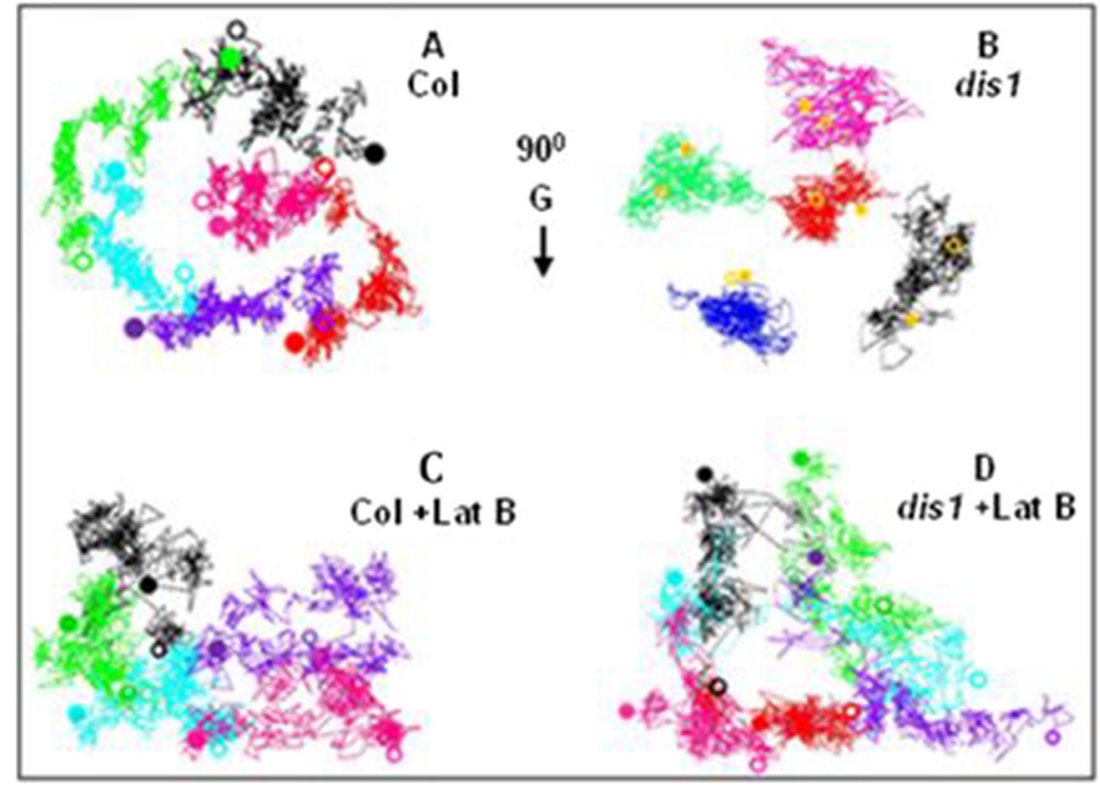

plants are also very different from that in the wild type plants. In dis 1 columella cells, the amyloplasts experience random small-step rattling motion confined within separate and relatively fixed regions without showing obvious cooperative cage-escape rearrangement (Fig. 2b), which is similar to the case in the jammed colloidal suspensions (Fig. 3d). The occasional large-step motion occurs mainly in the horizontal direction. It indicates that the cageconfinement in dis 1 mutant is much stronger than that in wild type plants. Interestingly, after Lat B treatment, the amyloplasts in both $\mathrm{Col}$ and dis 1 columella cells showed similar enhanced random diffusion with larger displacements and more homogeneous spatial distribution (Fig. 2c, d). It is similar to the case in dilute colloidal suspensions with weak or little cage-effect (Fig. 3d).
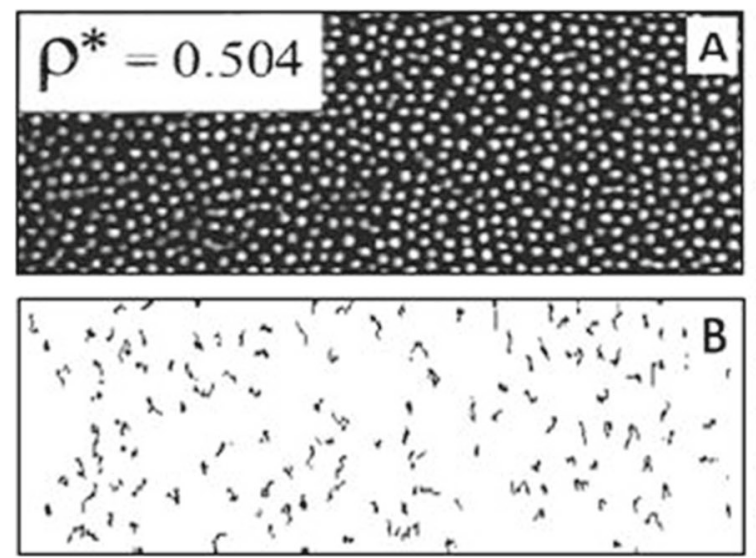

Fig. 3 Movement trajectories in colloidal suspensions a Optical microscopic image of the two-dimensional highly dense colloidal spheres with area fraction $\rho *=0.504$. b-d The typical movement

\section{Mean-Square-Displacements for Quantitative Measurement of Amyloplast Dynamics}

The diffusive dynamics of a single particle is a reflection of the interaction with the surrounding local environments which largely influences the collective sedimentation movements (Squires and Mason 2009). Hence, a quantified measurement of the diffusive dynamics and collective movements of amyloplasts is required to clarify the interaction with actin cytoskeleton and other unknown components. The diffusive dynamics of amyloplasts can be quantitatively characterized by the mean square displacements (MSD) against the time interval $t$. MSD was also introduced to describe the amyloplast movement in plant shoots (Toyota et al. 2013), but the diffusive motion was
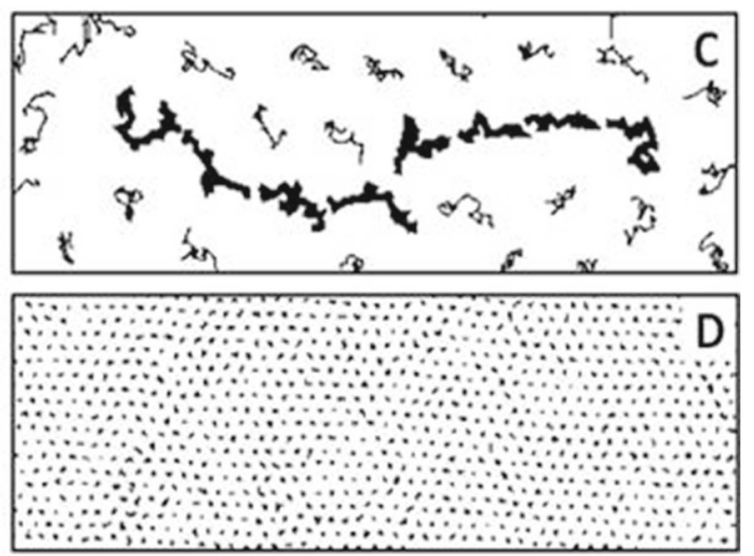

trajectories of colloidal spheres in the suspension with area fraction $\mathbf{b}$ $\rho=0.165$, c $\rho=0.504$ and $\mathbf{d} \rho=0.831$. These figures were cited from Marcus et al. (1999) 


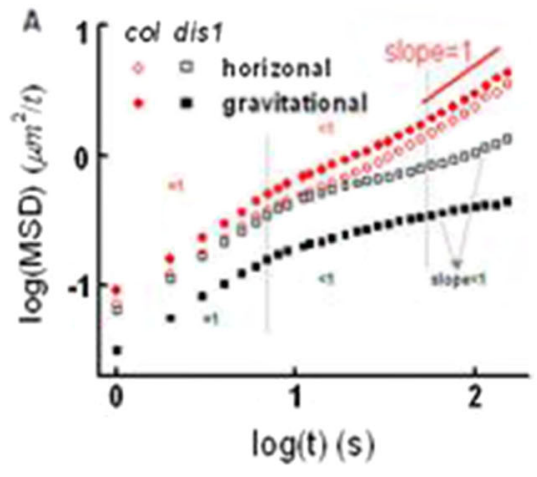

Fig. 4 Diffusive motions of the amyloplasts in horizontal and gravitational directions and in the two-dimensional colloidal suspensions characterized by typical $\log -\log \operatorname{MSD}(t)$. a Untreated Col and dis1 displayed distinct slopes at different time scales. b No differences

not analyzed. The red symbols in Fig. 4a shows the log$\log$ plot of $M S D(t)$ averaged from all amyloplasts in the columella cells of a wild type plant. It can distinctively divide into three time regimes, which is the characteristic behavior of a cage-diffusion process in dense colloidal suspension (red symbols in Fig. 4c) (Hunter and Weeks 2012; Zheng et al. 2011; Weeks et al. 2000). (1) In the initial short-time regime, the $\log -\log M S D(t)$ has a unit slope (i.e. a linear $M S D$ increase against time), indicating a free Brownian diffusion with the random Gaussian distribution of particle displacements $x(t)$ and $y(t)$ within a certain time $t$ (Marcus et al. 1999; Zheng et al. 2011). It reflects the random Brownian rattling motion of a particle before experiencing the surrounding cage. (2) In the intermediatetime regime, $M S D(t)$ develops a plateau with a slop $<1$, reflecting the encounter with the cage confinement imposed by surrounding circumstances and a crossover to subdiffusive motion. In this regime, the particle displacements deviate from Gaussian distribution (Fig. 5). It is also similar to between Lat B-treated $\mathrm{Col}$ and disl. c MSDs for colloidal suspensions with different area fractions taken from Zheng et al. (2011). The data was obtained from the statistics of amyloplasts in a single columella cell at the second layer for each type of plant

the non-Gaussian distribution of particle displacements in dense colloidal systems due to the excessive fraction of in-cage small-step rattling motion and occasionally largestep cooperative motion for escaping from the cages. (3) In the longtime regime, the $M S D(t)$ returns to a unit slope, reflecting the escape from cages and the regain of diffusive motion much slower than the short-time diffusion. The longtime diffusion corresponds to the collective rearrangement of particles with random displacements due to the random direct collisions.

The $\log -\log M S D(t)$ s of amyloplasts in the dis1 mutant (open black symbols in Fig. 4a) show a subdiffusive plateau lower than that of Col without regaining the long-time diffusion. The $M S D(t)$ in gravity direction is much lower than that in horizontal direction. It means that the cageconfinement is so strong that the amyloplasts can hardly escape in gravity direction. By contrast, after Lat B treatment, the $\log -\log M S D s$ of amyloplasts in both wild type and dis 1 plants show a linear increase with unit slope with-
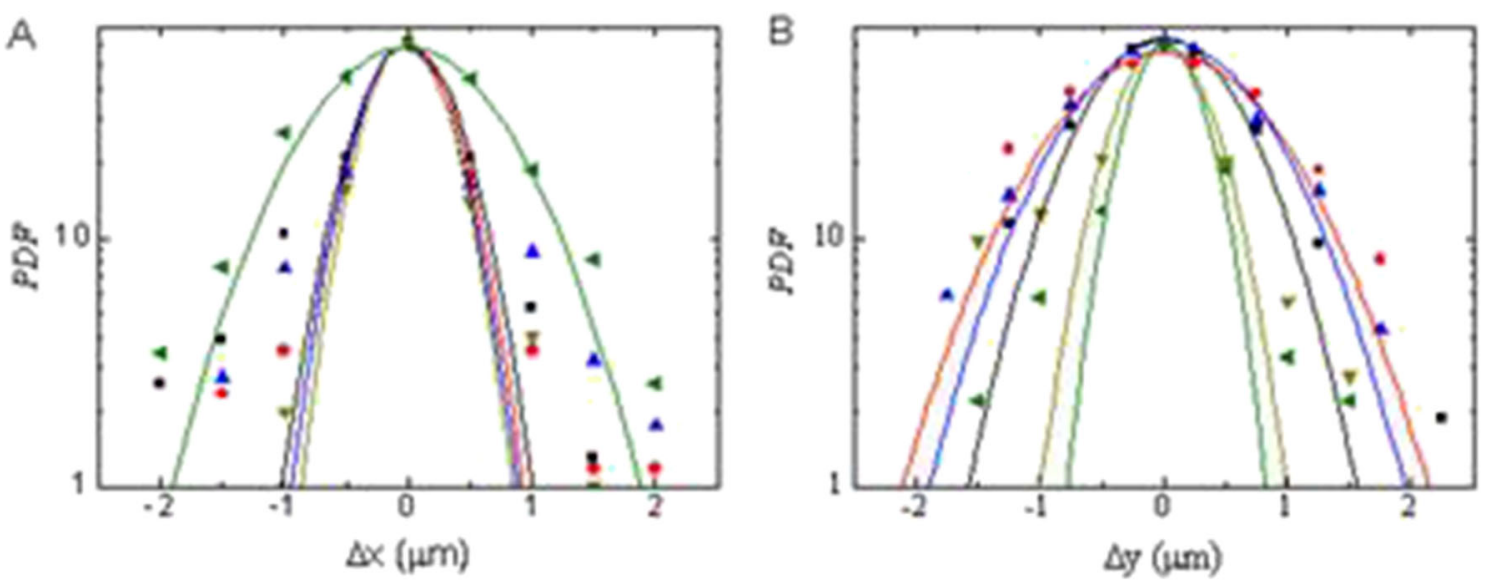

Fig. 5 Non-Gaussian probability distribution of the amyloplast displacements in the wild type columella cells during the cage-confinement diffusion in the horizontal (a) and gravitational (b) directions. Different colors denote different amyloplasts. The curves are Gaussian fittings 
Fig. 6 The spatial fluctuation of local apparent viscosity within gravity-sensing cells. a The nine subregions in the central columella cells. b-e The local apparent viscosity within the nine subregions in the central columella cells of different types of plants. The gray level represents the absolute magnitude of local apparent viscosity. The data was obtained from the statistics of amyloplasts in a single columella cell at the second layer for each type of plant

A Columella Cell subregion

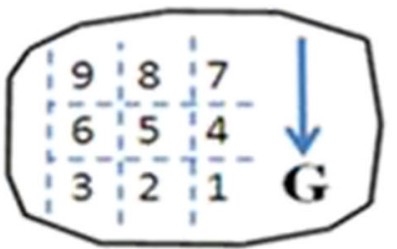

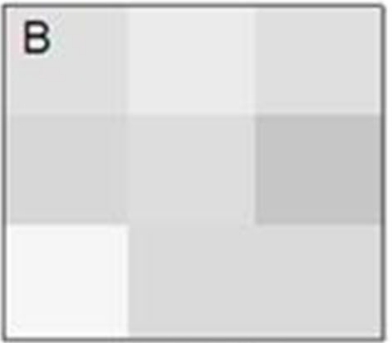

wild type

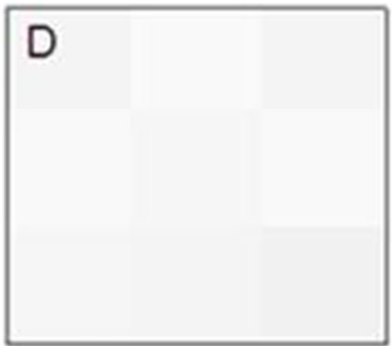

Lat B treated-col
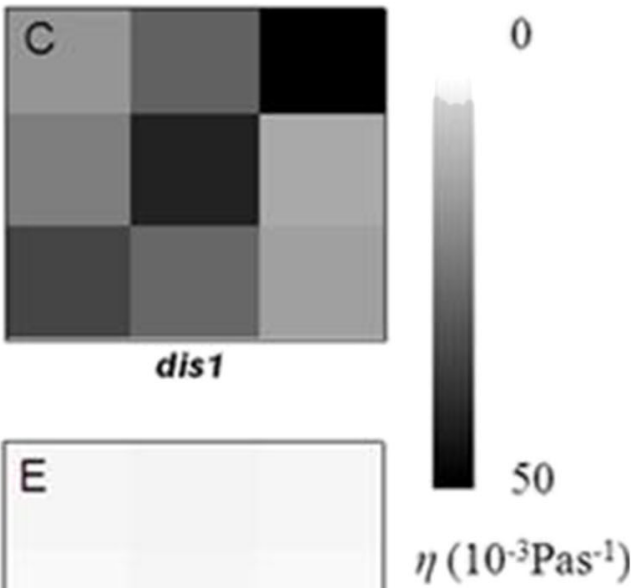

out an intermediate plateau regime (Fig. 4b). They are very similar to the MSD of diluted colloidal systems (black symbols in Fig. 4c), indicating weak or little cage-confinement in the collumela cells of Lat B treated plants.

\section{Microrheology Analysis and Local Apparent Viscosity in the Columella Cells}

We detected the local apparent viscosity in different subregions of the columella cells denoted in Fig. 6a by measuring the short-time diffusion coeffecient of amyloplasts within these regions in gravitational direction. The subregions were partitioned to compromise between the better description of the spatial heterogeneity in the columella cells and the better statistics in each subregion. In the columella cells of wild type plants, the local viscosity fluctuates from $\sim 2-15 \times$ $10^{-3} \mathrm{Pas}^{1}$ in different subregions (Fig. 6b), indicating the spatial heterogeneity of intracellular environment

The spatial fluctuation of local apparent viscosity in the columella cells of dis 1 plants is largely amplified to $\sim 5$ times of that in wild type plants (Fig. 6c). This result indicates stronger structural heterogeneity and stiffer cage confinement within the dis 1 columella cells than that of wild type plants. The local viscosity in columella cells are reduced to $\sim 50 \%$ of that of the untreated wild type plants (Fig. 6d, e). Therefore, Lat B treatment leads to nearly homogeneous intracellular environment and weak cageconfinement within columella cells and freely diffusive motion of amyloplasts. These greatly different sedimentation rates, trajectories and dynamics of amyloplasts and spatial distribution of local viscosity in the columella cells in wild type, disl and Lat Btreated plants are consistent with the different intracellular microenvironment resulted from different F-actin organizations in the columella cell.

Finally, we examined the possible effect of the amyloplast sedimentation on the biochemical signals and the final gravity response. It is thought that sedimentary amyloplasts cause strain deformation of unknown acceptor components that may activate biochemical signal, such as auxin We measured the 35S::DII-VENUS-N-N7 signal ratio between the upper and lower sides cells to monitor the auxin asymmetry and the final root curvatures at different times. Interestingly, we discovered that the inverse mean apparent viscosity $1 / \eta_{\text {mean }}^{\mathrm{B}}$ in the vicinity of the new cell bottom wall of the reoriented roots showed the linear relation with the DIIVENUS ratio and the root curvature angle as shown in Fig. 7a, b. Here, $\eta_{\text {mean }}^{\mathrm{B}}$ was averaged over the subregions 1-3 in the columella cell illustrated in the inset of Fig. 5a which 

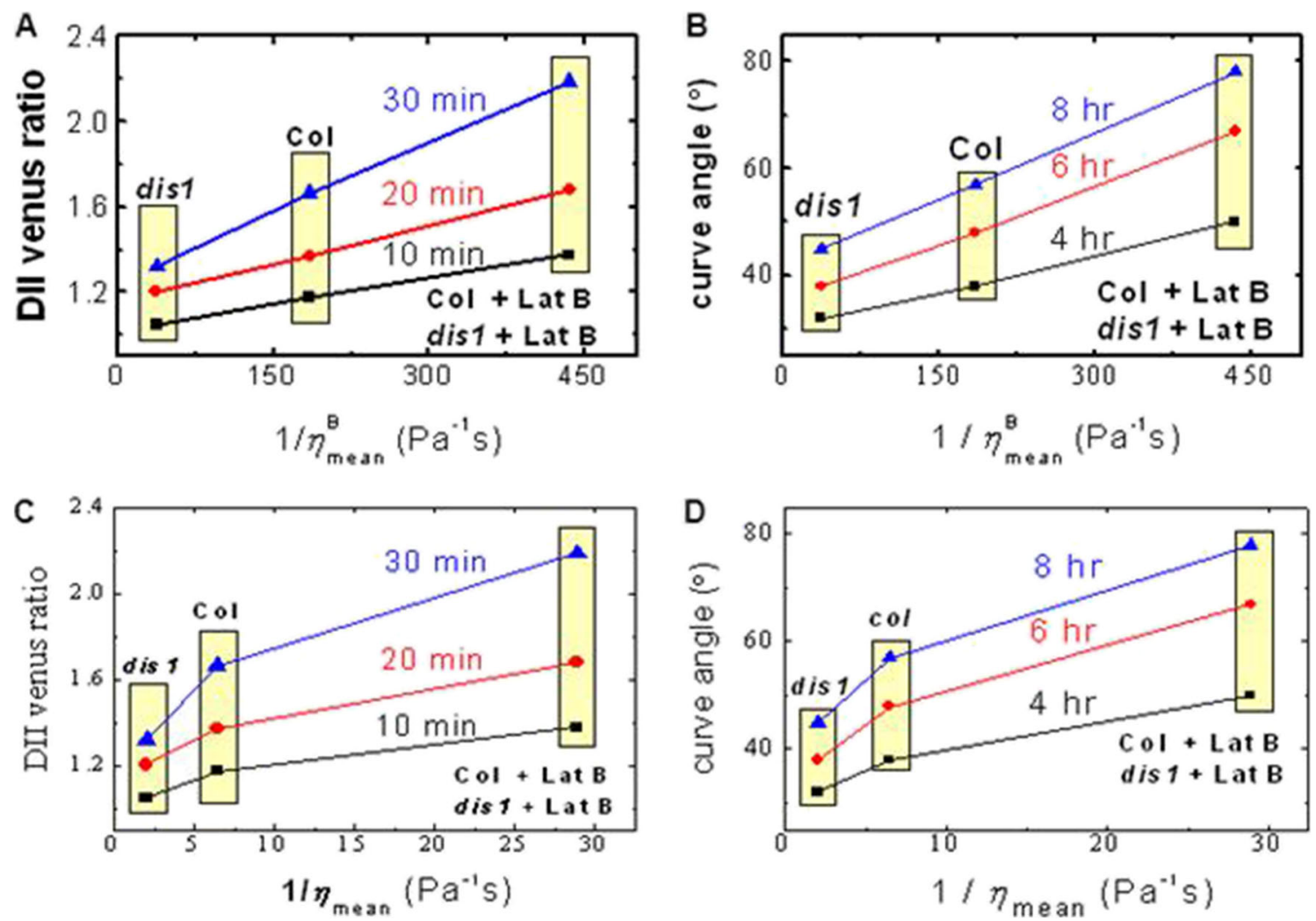

Fig. 7 The linear relation between DII-VENUS ratios (a) or root curvature angle (b) and the inverse mean viscosity $1 / \eta_{\text {mean }}^{\mathrm{B}}$. DII-VENUS ratios are measured between the upper and lower sides of lateral root cap cells adjacent to the columella cells at different times after 90 degree reorientation of the plants. $\eta_{\text {mean }}^{\mathrm{B}} \eta$ is averaged over subregions

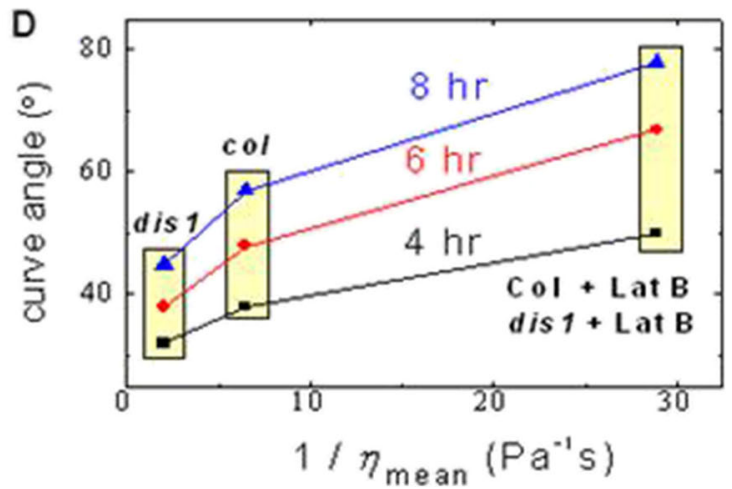

within the columella cells. $\mathbf{c}$ and $\mathbf{d}$ are the same as (a) and (b) respectively except for the replacement of $\eta_{\text {mean }}^{\mathrm{B}}$ by the average local apparent viscosity $\eta_{\text {mean }}$ within the entire columella cells. The data was obtained by averaging of 2-3 columella cells at the second layer in 3-5 typical plants (totally 9-15 cells) for each type of plants

are just adjacent to the cell bottom wall. The linear relation would not hold if $\eta_{\text {mean }}$ was averaged over the whole interior of columella cell (Fig. 7c, d). Since the mechanical effect is determined by the three main factors: magnitude, direction and loading point of the force, these results suggest that the mechanical stimulation of amyloplast sedimentation most probably takes effect in the vicinity of the new cell bottom walls, which is a local effect rather than a global average effect within the columella cells. Therefore, we proposed that such local effect of mechanical stimulation may be considered as an impulse (kinetic momentum) of the falling amyloplasts inducing the strain deformation of unknown components.

For an object falling slowly at nearly constant velocity, its velocity is inversely proportional to the drag force imposed by surrounding solution which can be measured by the viscosity. Here, $\eta$ reflects the cytoplasmic resistance dominated by actin cytoskeleton and $1 / \eta$ is usually called actin cytoskeleton fluidity. Hence, the impulse of the amyloplasts $L=m_{\text {mean }} \cdot v_{\text {mean }} \propto 1 / \eta_{\text {mean }}^{B}$ is an equivalent measurement of the strain deformation of the acceptor components,

where $M_{\text {mean }}$ and $v_{\text {mean }}$ are the mean mass and mean velocity of amyloplasts, respectively. These results reasonably suggest that the degree of biochemical and gravimorphogenetic responses should be closely related to the strength of mechanical stimulation. Furthermore, they also suggested the existence of a potential gravity-sensing mechanism that dictates a linear frustration effect of the actin cytoskeleton on the conversion of the mechanical stimulation of amyloplasts into gravitropic signals.

\section{Conclusion}

In this work, we studied the gravity sensing in the columella cells in plant roots by analyzing the diffusive dynamics and microrheology of amyloplasts during sedimentation. First, we found that the intracellular environments of the collumelar cells have the colloidal-like spatial heterogeneities. The amyloplasts in wild type plants perform cage-confinement diffusion and the string-like collective cage-breaking motion, which are the characteristic 
features in highly dense colloidal systems. By contrast, the cage-confinement effect on amyloplasts in dis1 and Lat B treated plants becomes stronger and weaker just as the case in the jammed and dilute colloidal suspensions. Second, the cage-effect and the spatial heterogeneities were quantitatively characterized by the local apparent viscosity obtained by using the in-vivo microrheology analysis. We found that the spatial fluctuation of the local apparent viscosity in dis1 and Lat B treated plants is about five times and a half of that in the wild type plants. These observations correspond to the moderate, strong and weak cage-confinement effect on alymloplasts in the wild type, dis 1 and Lat B-treated plants. We proposed that the different actin-cytoskeletal organizations should be responsible for the different intracellular microenvironments of the columella cells in these different types of plants, which determine the different dynamics during the amyloplast sedimentation. Finally, we found the linear relation between the inverse of average apparent viscosity near the cell bottom walls, the DII-VENUS ratio of the upper and lower lateral root cap and the root curvature. These observations reasonably suggest that the mechanical stimulation of amyloplast sedimentation most probably takes effect in the vicinity of the new cell bottom walls and the degree of biochemical and gravimorphogenetic responses should be closely related to the strength of mechanical stimulation.

Acknowledgments This work was supported by the National Basic Research Program of China (Grant No. 2011CB710902, 2011CB710901) and NSFC grants 11104286 and 11372314.

\section{References}

Band, L.R., Wells, D.M., Fozard, J.A., Ghetiu, T., French, A.P., et al.: Systems analysis of auxin transport in the Arabidopsis root apex. Plant Cell 26, 862-875 (2014)

Basu, D., Le, J., El-Essal Sel, D., et al.: DISTORTED3/SCAR2 is a putative arabidopsis WAVE complex subunit that activates the Arp2/3 complex and is required for epidermal morphogenesis. Plant cell 17, 502-524 (2005)

Blancaflor, E.B.: Regulation of plant gravity sensing and signaling by the actin cytoskeleton. Am. J. Bot. 100, 143-152 (2013)

Brunoud, G., Wells, D.M., Oliva, M., Larrieu, A., Mirabet, V., et al.: A novel sensor to map auxin response and distribution at high spatiotemporal resolution. Nature 482, 103-106 (2012)

Chaudhuri, O., Parekh, S.H., Fletcher, D.A.: Reversible stress softening of actin networks. Nature 445, 295-298 (2007)
Crocker, J.C., Valentine, M.T., Weeks, E.R., et al.: Two-point microrheology of inhomogeneous soft materials. Phys. Rev. Lett. 85, 888-891 (2000)

Hou, G., Kramer, V.L., Wang, Y.-S., et al.: The promotion of gravitropism in Arabidopsis roots upon actin disruption is coupled with the extended alkalinization of the columella cytoplasm and a persistent lateral auxin gradient. Plant J. 39, 113-125 (2004)

Hunter, G.L., Weeks, E.R.: The physics of the colloidal glass transition. Rep. Prog. Phys. 75, 066501 (2012)

Le, J., El-Assal Sel, D., Basu, D., Saad, M.E., Szymanski, D.B.: Requirements for Arabidopsis ATARP2 and ATARP3 during epidermal development. Curr. Biol. 13, 1341-1347 (2003)

Leitz, G., Kang, B.-H., Schoenwaelder, M.E.A., Staehelin, L.A.: Statolith sedimentation kinetics and force transduction to the cortical endoplasmic reticulum in gravity-sensing Arabidopsis columella cells. Plant Cell 21, 843-860 (2009)

Levine, A.J., Lubensky, T.C.: One- and two-particle microrheology. Phys. Rev. Lett. 85, 1774-1777 (2000)

Marcus, A.H., Schofield, J., Rice, S.A.: Experimental observations of non-Gaussian behavior and stringlike cooperative dynamics in concentrated quasi-two-dimensional colloidal liquids. Phys. Rev. E 60, 5725-5736 (1999)

Mizuno, D., Tardin, C., Schmidt, C.F., Mackintosh, F.C.: Nonequilibrium mechanics of active cytoskeletal networks. Science 315, 370373 (2007)

Morita, M.T.: Directional Gravity Sensing in Gravitropism. Annu. Rev. Plant Biol. 61, 705-720 (2010)

Palmieri, M., Kiss, J.Z.: Disruption of the F-actin cytoskeleton limits statolith movement in Arabidopsis hypocotyls. J. Exp. Bot. 56, 2539-2550 (2005)

Perbal, G., Driss-Ecole, D.: Mechanotransduction in gravisensing cells. Trends Plant Sci. 8, 498-504 (2003)

Squires, T.M., Mason, T.G.: Fluid mechanics of microrheology. Annu. Rev. Fluid Mech. 42, 413 (2009)

Swarup, R., Kramer, E.M., Perry, P., et al.: Root gravitropism requires lateral root cap and epidermal cells for transport and response to a mobile auxin signal. Nat. Cell Biol. 7, 1057-1065 (2005)

Toyota, M., Ikeda, N., Sawai-Toyota, S., et al.: Amyloplast displacement is necessary for gravisensing in Arabidopsis shoots as revealed by a centrifuge microscope. Plant J. 76, 648-660 (2013)

Vanneste, S., Auxin, F.J.: A trigger for change in plant development. Cell 136, 1005-1016 (2009)

Waigh, T.A.: Microrheology of complex fluids. Rep. Prog. Phys. 68, 685-742 (2005)

Weeks, E.R., Crocker, J.C., Levitt, A.C., Schofield, A., Weitz, D.A.: Science 287, 627 (2000)

Wirtz, D.: Particle-tracking microrheology of living cells: principles and applications. Annu. Rev. Biophys. 38, 301-326 (2009)

Zheng, Z., Han, Y.: Self-diffusion in two-dimensional hard ellipsoid suspensions. J. Chem. Phys. 133, 124509 (2010)

Zheng, Z., Ni, R., Wang, F., Dijkstra, M., Wang, Y., Han, Y.: Structural signatures of dynamic heterogeneities in monolayers of colloidal ellipsoids. Nat. Commun. 5, 3829 (2014)

Zheng, Z., Wang, F., Han, Y.: Glass transitions in quasi-twodimensional suspensions of colloidal ellipsoids. Phys. Rev. Lett. 107, 065702 (2011) 